\title{
Comparative Study of Energy Consumption for Wireless Sensor Networks based on Random and Grid Deployment Strategies
}

\author{
Monica \\ Department of Computer Science and Engineering \\ National Institute of Technology, \\ Jalandhar, Punjab, India
}

\author{
Ajay K Sharma \\ Department of Computer Science and Engineering \\ National Institute of Technology, \\ Jalandhar, Punjab, India
}

\begin{abstract}
Deployment of the nodes in a wireless sensor network to satisfy continuous sensing with extended network lifetime while maintaining uniform coverage in the deployment region is the major challenge in wireless sensor networks. Various architectures and node deployment strategies have been developed for wireless sensor network, depending upon the requirement of application. Node deployment in wireless sensor network is application dependent and can be either deterministic or randomized. In this paper we present the relation between the energy consumption and the deployment strategy. We present a comparative investigation of the random and grid deployment strategy of the sensor nodes in wireless sensor networks based on energy consumption. We have also compared the energy consumption by the few commercially used chipsets based on two deployment strategies.
\end{abstract}

\section{Keywords}

Wireless Sensor Networks, Random deployment, Grid Deployment, Chipsets

\section{INTRODUCTION}

The researchers are attracted towards the idea of Wireless Sensor Networks (WSNs) due to wide range of potential applications that it offers such as biological detections, home security, environment detection and monitoring, habitat monitoring etc. [1]. Sensor nodes that are used to form a WSN are normally operated with small battery that have small amount of energy. Moreover, sensor nodes are usually deployed in dangerous and inhospitable regions which make the replacement or the recharge of the battery difficult or impossible. Therefore, in WSNs reducing energy consumption of each sensor node is one of the important issues to prolong the network lifetime [2]. In this paper we investigate the energy consumption based on two deployment strategies for the few commercially used chipsets.

Sensor networks are usually deployed in a sensing field to collect useful information from it. Deployment is concerned with setting up an operational sensor network in a real-world environment. Usually the deployment of sensor network is a labor- intensive and cumbersome task as the real-world influences trigger bugs or degrade performance in a way that has not been observed during pre-deployment. This is due to the reason that the functions of the sensor network is strongly influenced by the real world which controls the output of the sensors [3]
Deploying sensors to provide complete area coverage is another essential design problem in many wireless sensor network (WSN) applications. Mainly three alternative deployment approaches have been proposed in literature. One among them is application-specific deterministic deployment, another is random deployment and the third one is grid based (also known as pattern-based) deployment [4-5]. In deterministic deployment, the sensor nodes are placed deliberately in the required region. This type of deployment is suitable only for small-scale applications. Non-deterministic deployment is scalable to largescale applications or hostile environments. In this type of deployment, the sensor nodes are thrown randomly to form a WSN. However, it could be very expensive since excess redundancy is required to overcome uncertainty. Grid-based deployment is an attractive approach for moderate to large-scale coverage-oriented deployment due to its simplicity and scalability. In this research, we concentrate on the amount of energy consumed by the sensor nodes while they are deployed in random and grid fashion. A comparison of the energy consumption for the chipset TR1001, CC1000 and CC1010 is also investigated for both random and grid deployment.

Previous research has explored the properties of grid-based deployment in the ideal circumstance where individual sensors are placed exactly at grid points. However, in practice, it is often infeasible to guarantee exact placement due to various errors, including misalignments and random misplacement [6]. Literature has proposed various algorithms which helps reducing the energy consumption for the different deployment strategies. Here, in this paper, make a comparison of the energy consumption based on two different deployment strategies for the few commercially available chipsets.

The rest of the paper is organized as follows. Section 2 throws some light on the related research work. Section 3 focuses on the deployment considerations. Section 4 discusses the network setup and results and finally the conclusion and future scope is analyzed in Section 5.

\section{RELATED WORK}

In 2002 Andrew Howard et al. [7] considered the problem of deploying a mobile sensor network in an unknown environment. In the paper, a potential-field-based approach to deployment has been presented. The fields are constructed such that each node is repelled by both obstacles and by other nodes, thereby forcing the network to spread itself throughout the environment. The approach is both distributed and scalable. 
In 2005, Ali Iranli et al., [8] investigated and developed energy sufficient strategies for deployment of WSN for the purpose of monitoring some phenomenon of internet in the coverage region. In this paper, the operational advantages of two level hierarchical architecture over flat network architecture have been studied. The paper formulated and solved the problem of assigning positions and initial energy levels to micro-servers and concurrently partitioning the sensors into clusters assigned to individual micro-servers so as to maximize the monitoring lifetime of the two-level WSN subject to total energy budget. Richard Tynan et al., in 2005 [9], proposed a methodology for the rapid development of a Multi-Agent System (MAS) for WSNs that allows a comprehensive testing and debugging, a luxury not available on current WSN devices. The methodology allowed for the verification of the correctness of the algorithm before deployment.

Chih-Yung Chang et al., in 2005 [10], proposed a Zone-Based Broadcasting protocol for WSNs. The article proposed an efficient broadcasting protocol to reduce the number of the sensor nodes that forward the query request, hence improves the packet delivery rate and saves bandwidth and power consumption. Sensor nodes that received the query request will dynamically transfer the coordinate system according to the zoneID of the source node and determines whether it would forward the request or not in a distributed manner. Compared with the CBM and traditional flooding operations, experimental results show that the proposed zone-based broadcasting protocol decreases the bandwidth and power consumption, reduces the packet collision and achieves high success rate of packet broadcasting.

Jinghao Li et al., in 2006 [11], analyzed the deployment challenges and then proposed a planning and deployment platform for WSNs, namely POWER, to resolve these problems. POWER has three main parts, network deployment, simulation, and performance evaluation and optimization. Simulation is the foundation of the POWER. Lifetime is the most important performance evaluation metrics. The target of the POWER is to supply an integrated and optimal deployment solution for an actual application.

Chih-yung Chang et al., in 2006 [12], proposed an efficient node placement and topology control protocols to balance the power consumption of sensor nodes. Firstly, a virtual tree topology is constructed based on Grid-based WSNs. Then two node placement techniques, namely Distance-based and Density-based deployment schemes are proposed to balance the power consumption of sensor nodes. Finally, extension of the proposed protocols are made from a Grid-based WSN to a randomly deployed WSN, making the developed energy-balanced schemes can be generally applied to randomly deployed WSNs. Simulation results reveal that the developed protocols can efficiently balance each sensor node's power consumption and prolong the network lifetime in both Grid-based and randomly deployed WSNs.

Matthias Ringwald and Kay Romer in 2007 [3] addressed the problems encountered during the deployment of sensor networks. Deployment is a labor-intensive and cumbersome task as environmental influences often degrade performance or trigger bugs in the sensor network that could not be observed during lab tests. In this paper, firstly, existing sensor networks has been investigated to identify and classify typical problems that have been encountered during deployment. Secondly, an investigation has been made on whether and how the existence of these problems could be detected by means of passive inspection, where messages exchanged in the sensor network are overheard and analyzed such that modification of the sensor network is not required. It has also been shown that how passive inspection could be implemented in a practical tool. Pradnya Gajbhiye and Anjali Mahajan, in 2008 [13], presented the survey of state-ofthe-art of architecture and node deployment in wireless sensor network. They presented the characteristics of the environment in which the sensor networks may deploy. Node deployment in wireless sensor network is application dependent and can be either deterministic or randomized. But in both the cases coverage of interested area is the main issue. Routing protocols for wireless sensor network were also proposed

\section{DEPLOYMENT CONSIDERATION}

There are certain pertinent issues relating to the sensor network deployment. An essential requirement is the presence of at least one data sink node, namely gateway, at which data transmitted from the distributed sensors converges. As such, it is preferable for the gateway typically to have higher energy and processing capabilities. The case of a large scale sensor networks may necessitate the deployment of multiple gateways. Adequate coverage for the area of interest requires placement of the gateways in the manner that minimizes disparity between the sizes of the subnetwork covered by each gateway.

\subsection{Random Deployment}

Here, we considered random deploying of sensors. It means there place in the network is not considered previously. Random deployment of sensor nodes in the physical environment may take several forms. It may be a one-time activity where the installation and use of a sensor network are strictly separate actions. Or, it may be a continuous process, with more nodes being deployed at any time during the use of the network; for example, to replace failed nodes or to improve the coverage area at certain locations $[1,14]$. Figure 1 (a) shows the deployment of the sensor nodes in random fashion for a wireless sensor network.

- Randomized sensor deployment is quite challenging in some respects, since there is no way to configure a priori the exact location of each device

- Additional post-deployment self-configuration mechanisms are required to obtain the desired coverage and connectivity

- In case of a uniform random deployment, the only parameters that can be controlled apriori are the numbers of nodes and some related settings on these nodes, such as their transmission range.

\subsection{Grid Deployment}

There are three types of grid-based deployment corresponding to three regular shapes which can tile a plane without holes, namely, hexagon, square and equilateral triangle. Grid-based deployment is conducted by dropping sensors row-by row using a 
moving carrier. Figure 1 (b) shows the deployment of the sensor nodes in grid fashion for a wireless sensor network. The time interval between consecutive droppings is controlled to achieve the desired distance. However, often this ideal deployment is not realistic due to placement errors [15]. In the unreliable sensor grid model, $\mathrm{n}$ nodes are placed on a square grid within a unit area, with a certain probability that a node is active (not failed), and a defined transmission range of each node.

- Adding nodes to ensure wireless connectivity is a challenging issue, particularly when there are location constraints in a given environment that dictate where nodes can or cannot be placed

- If the number of available nodes is small with respect to the size of the operational area and required coverage, a balance between sensing and routing nodes has to be optimized

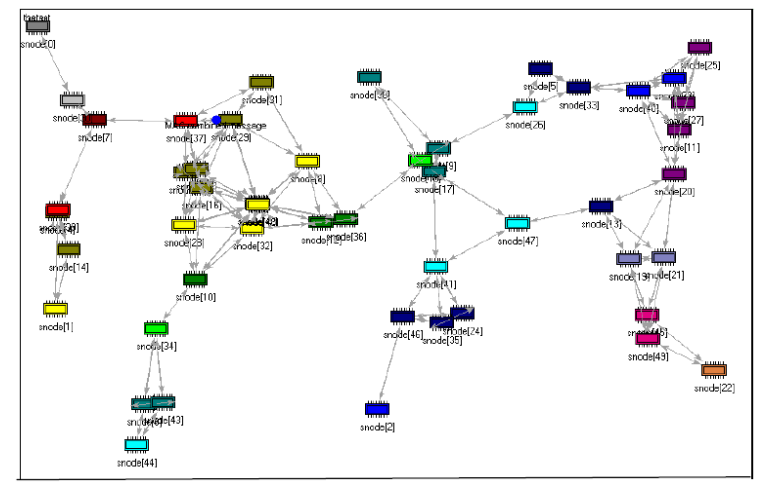

(a)

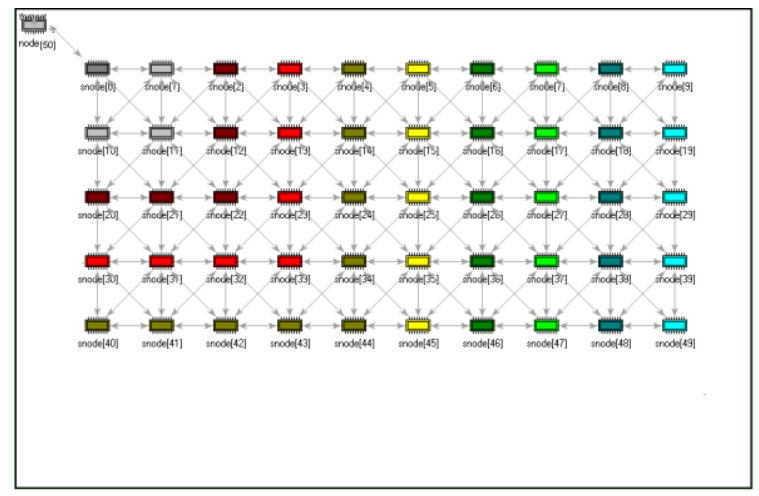

(b)

FIGURE 1: Deployment strategies for sensor nodes in wireless senor network (a) Random (b) Grid

\section{RESULTS AND DISCUSSIONS}

In our simulation, we use a rectangular space of $800 \times 500$ centimeters as the sensing area, one of the corners being fixed at origin. We have chosen to work with two different scenarios, one with random deployment of sensor nodes and another with grid deployment of sensor nodes. Each of the scenarios consists of 50 sensor nodes including one gateway. The node transmitting range allows communication with direct neighbors only. The simulation is aimed to evaluate the energy consumed by the sensor node for a particular type of deployment strategy. The attempts are also made to find out the difference in the energy consumption for the two different deployment strategies for few commercially available chipsets. Each graph has been obtained by running 7 independent simulations of 20 minutes each.

CASE 1: Energy consumed during random deployment Figure 2 (a), (b) and (c) shows the energy composition during random deployment of the chipsets TR1001, CC1000 and CC1010 respectively for the transmission, reception, switching and sleep mode.

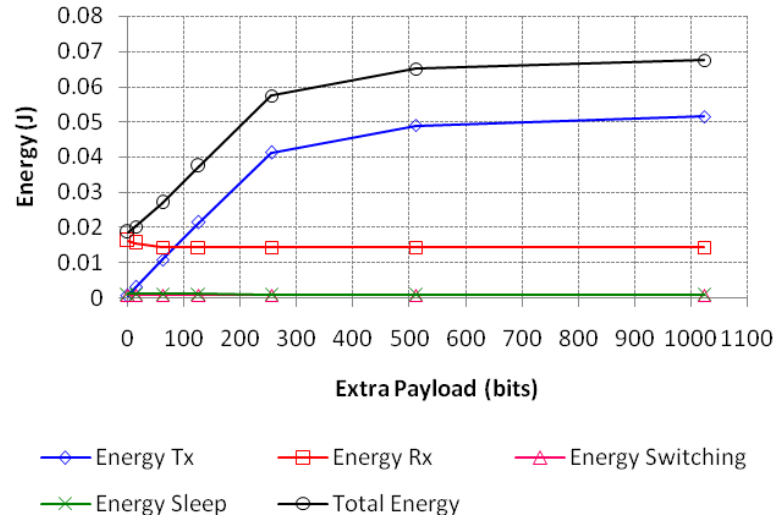

(a)

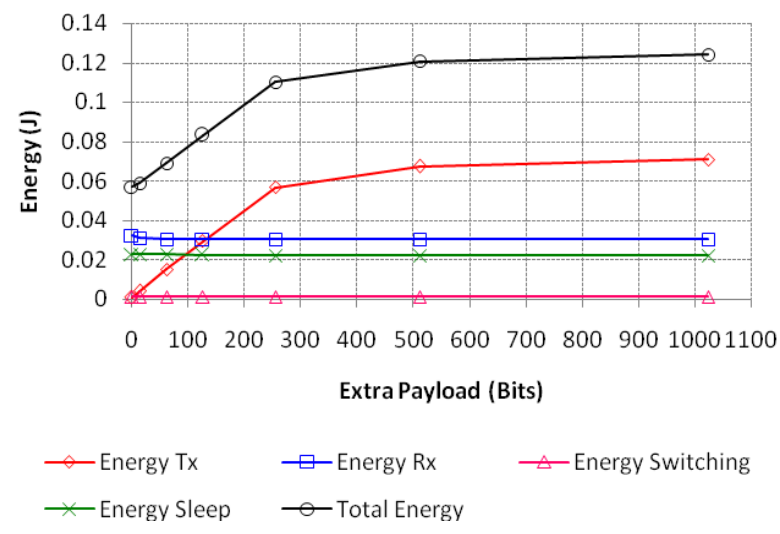

(b)

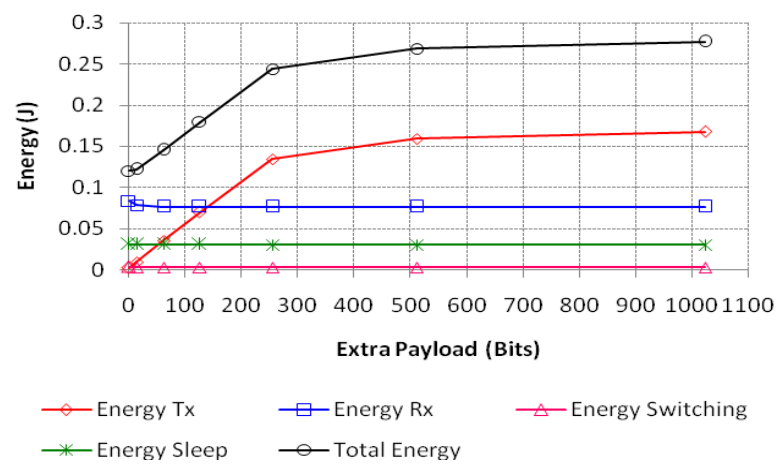

(c)

FIGURE 2: Sensor Node Energy versus Extra Payload for random deployment (a) TR1001, (b) CC1000 and (c) CC1010 
CASE 2: Energy consumed during grid deployment

Figure 3 (a), (b) and (c) shows the energy consumption for the grid deployment of sensor nodes in the WSN for the chipsets TR1001, CC1000 and CC1010 respectively. The comparisons of the individual energies consumption modes for grid and random distribution have been shown in further graphs.

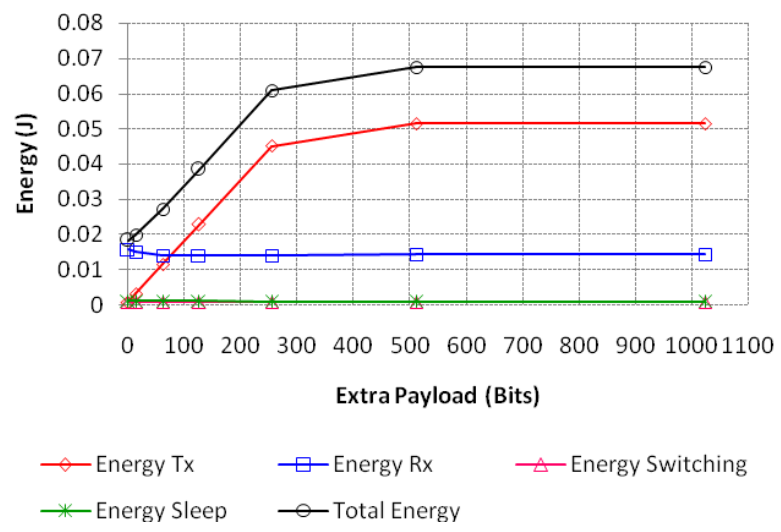

(a)

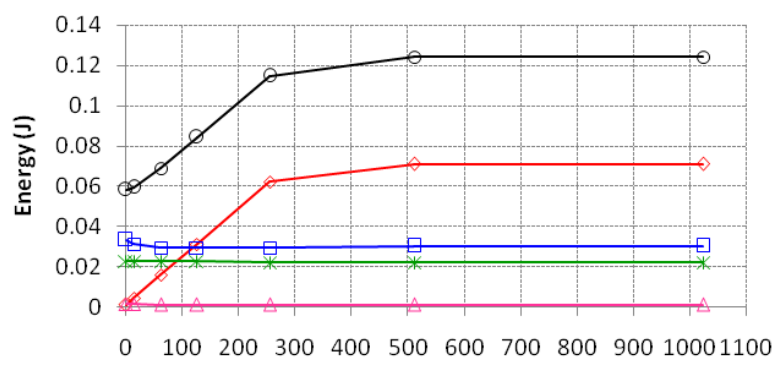

Extra Payload (Bits)

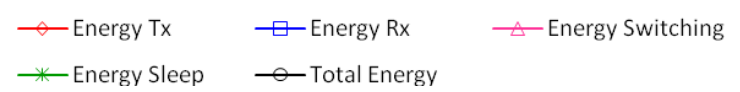

(b)

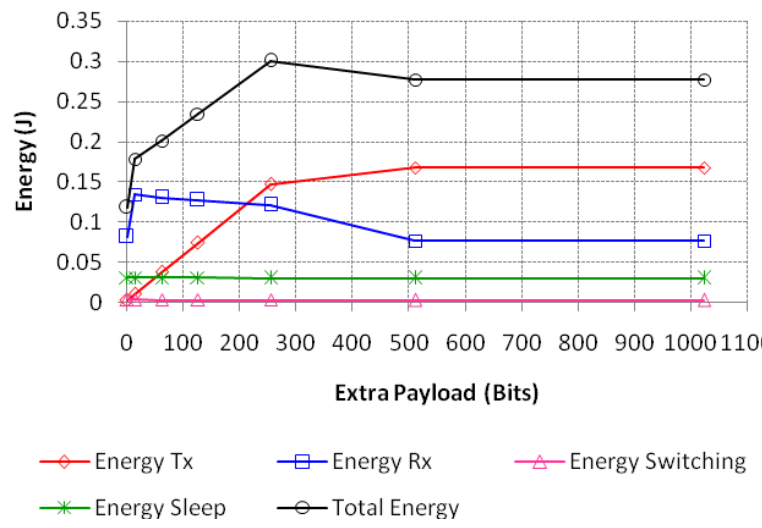

(c)

FIGURE 3: Sensor Node Energy versus Extra Payload for grid deployment (a) TR1001, (b) CC1000 and (c) CC1010
CASE 3: Energy consumed by chipset TR1001 for random vs. grid deployment

Figure 4 shows a comparison of energy consumption for random and grid deployment by the chipset TR1001 for different energy consumption modes. Figure 4 (a) shows the comparison of energy consumption during data transmission for the random and grid deployment for the chipset TR1001. It is evident from the graph that for the initial and final value of the extra payload the energy consumption for both the random and grid deployment is same. There is variation in the energy consumptions in between the initial and the final values of the extra payload. It is depicted from the graph that energy consumption is more in case of grid deployment than that of random deployment.

Figure 4 (b) shows the energy consumption during the reception of data for the random and grid deployment for the chipset TR1001. It can be seen from the graph that initially the energy consumed by the random deployment is more than that of grid deployment and then for the extra payload of 511 upto 1024, the values of the energy consumption became stable and coincides to a common point.

Figure 4(c) shows the comparison of energy consumed during switching for the random and grid deployment for the chipset TR1001. The figure depicts that at extra payload of $1 \mathrm{bit}$, the switching energy for random and grid is $0.000762 \mathrm{~J}$ and $0.000789 \mathrm{~J}$.

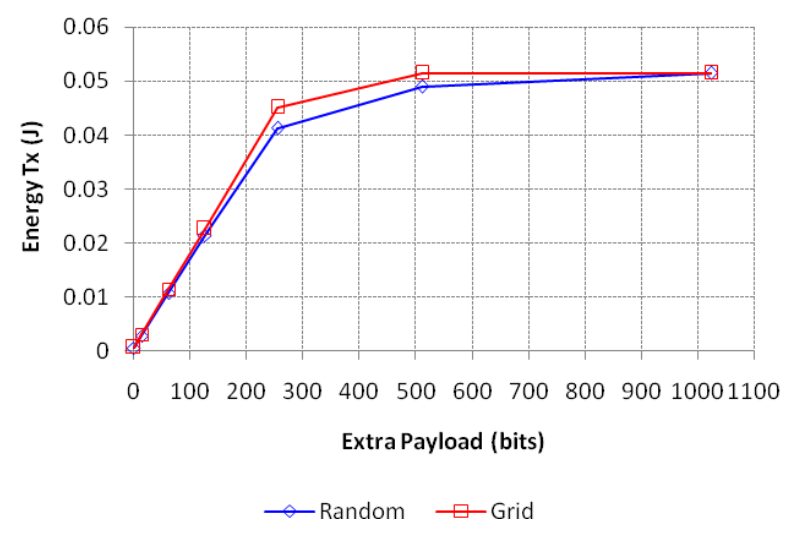

(a)

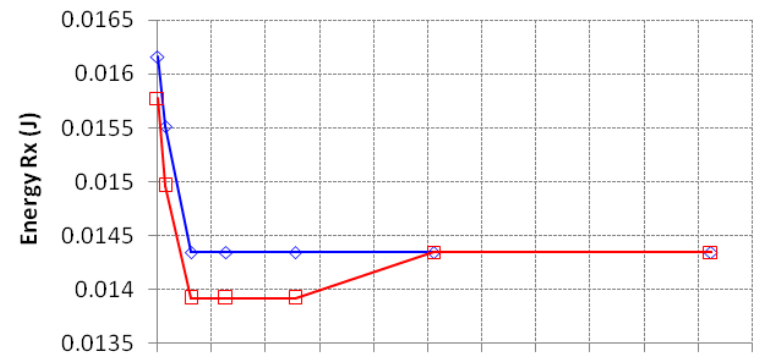

$0 \quad 10020030040050060070080090010001100$

Extra Payload (bits)

$\smile$ Random $\square$ Grid

(b) 


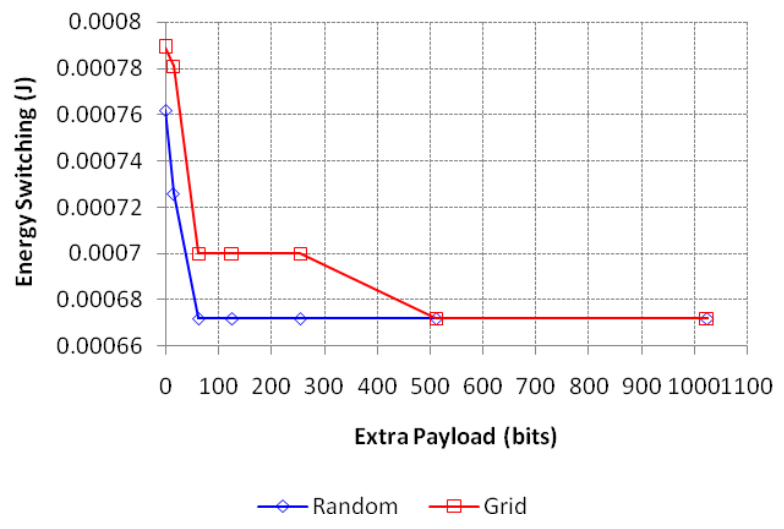

(c)

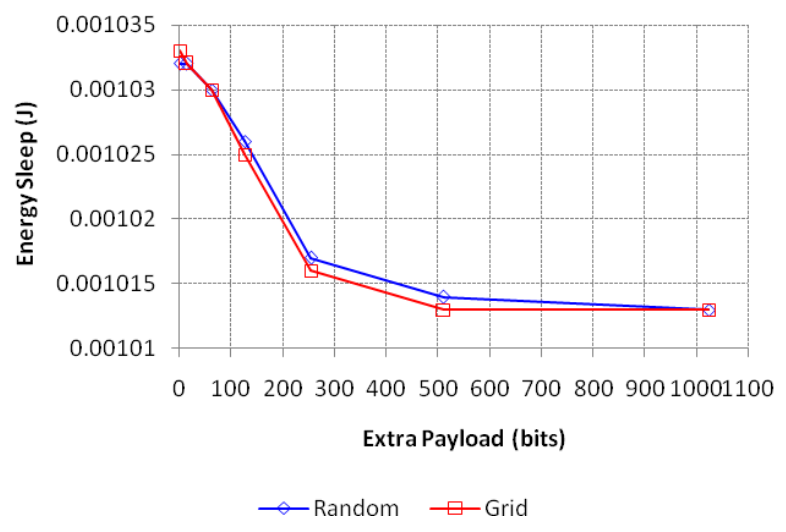

(d)

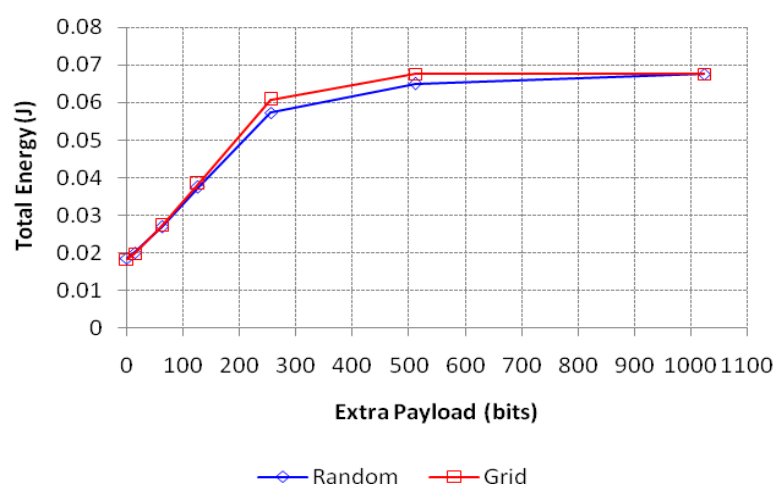

(e)

FIGURE 4: Comparison of random and grid deployment for TR1001 (a) Energy Tx, (b) Energy Rx, (c) Energy Switching, (d) Energy Sleep, (e) Total Energy

The switching energies for both the deployments shows a sharp decrease upto extra payload of 63 bits where its value decrements to $0.000672 \mathrm{~J}$ and $0.0007 \mathrm{~J}$ for random and grid respectively. After the extra payload of 511 bits, the switching energy values become smooth for both the deployments. Overall we can see that in case of switching from one state to another, random deployment consumes less energy than grid deployment.
Figure 4 (d) shows the energy consumed by the chipset TR1001 during sleep mode for the two deployment strategies. It is very much clear from the figure that the energy consumption during sleep for grid deployment is less than that of random deployment. At the extra payload of 1 bit, the sleep energy consumed by a node which is deployed randomly is $0.001032 \mathrm{~J}$ and for the node which is deployed in grid fashion is $0.001033 \mathrm{~J}$. The energy consumption falls down sharply for both the deployments upto an extra payload of 255 bits, and after that they show a gradual decrease upto the extra payload of 1023 . This downward trend of the sleep energy is due to the fact that as the payload increases, the node takes more time to transmit, and hence gets less time to sleep. And hence the sleep energy decreases with the increasing payload.

In Figure 4 (e), a comparison of the total energy consumed by a sensor node TR1001, for the random and grid deployment has been presented. It is observed that the grid deployment consumes more total energy than that of random deployment. The figure also depicts that for the extra payload of 1 bit upto 127 bits, the total energy is same for both the random and grid deployment after which the energy consumption by grid starts increasing. As the extra payload value increases beyond 511 bits, the difference between the energy consumption for the two deployments reduces and at the extra payload of 1023, the value of energy consumption coincides to a single value i.e. $0.077743 \mathrm{~J}$.

\section{CASE 4: Energy consumed by chipset $\mathrm{CC1000}$ for random vs. grid deployment}

Figure 5 shows a comparison of energy consumption for random and grid deployment by the chipset CC1000 for different energy consumption modes. Figure 5 (a) shows the energy required for transmission by for chipset CC1000 for random and grid deployment. For the initial value of extra payload, the energy Tx is almost negligible for both the distributions. As the extra payload increases the Energy Tx also increases. It is evident from the figure that the energy consumption during transmission by the grid deployment is greater than that of the random deployment.

The Figure 5 (b) shows a comparison of energy consumed during the reception for the chipset $\mathrm{CC} 1000$ for random and grid deployment. For extra payload of 1 bit, the energy Rx for a sensor node placed in a randomly deployed network and a grid network is $0.03208 \mathrm{~J}$ and $0.033215 \mathrm{~J}$. As the extra payload increases from 1 bit to 63 bits, the energy Rx for the sensor node in both random and grid deployment decreases sharply and reaches to $0.030243 \mathrm{~J}$ and $0.029354 \mathrm{~J}$ after that the energy Rx for random deployment remains smooth. The Figure 5 (b) also depicts that sensor nodes deployed in grid fashion consumes less energy for reception while the nodes deployed in random fashion consumes more energy.

Figure 5 (c) shows the energy consumed during transition from one state to another by the chipset $\mathrm{CC} 1000$ for the random and grid deployment. It is evident from the figure that the sensor nodes deployed in grid fashion consumes more energy than the sensor nodes deployed in random fashion during switching from one state to another. Also for low value of extra payload, the switching energy is maximum, and then its shows a sudden downfall upto the 63 bits of extra payload, after which it 
becomes stable for both types of deployment. At the extra payload of 511 bits, the values of switching energy becomes equal for both types of deployment and remains constant for the increasing values of extra payload.

Figure 5 (d) shows the comparison of energy consumed during sleep for the chipset CC1000 for the random and grid deployment. The figure indicates that the energy sleep for the sensor node in grid deployment is less than the node in the random deployment.

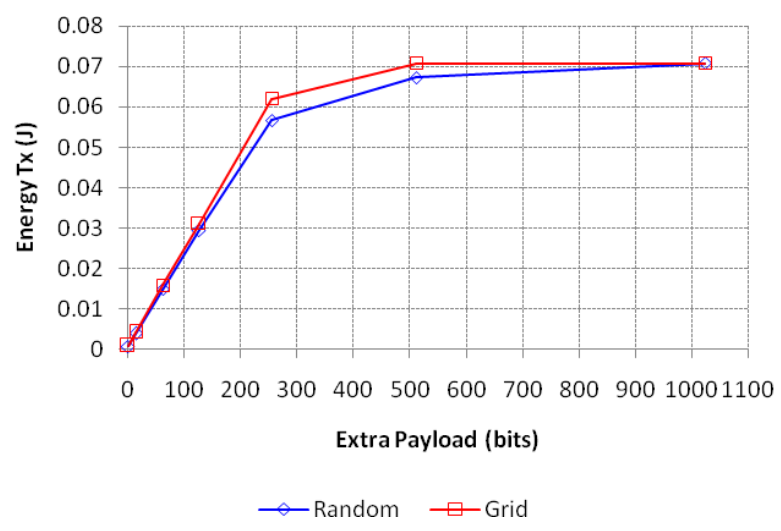

(a)

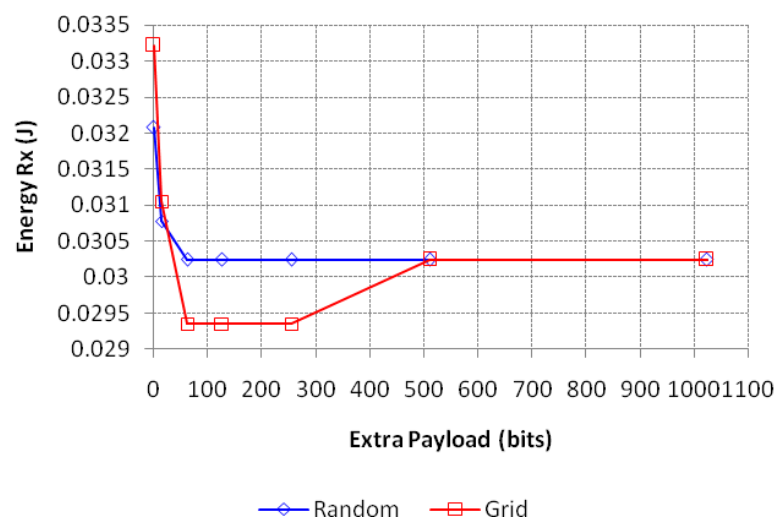

(b)

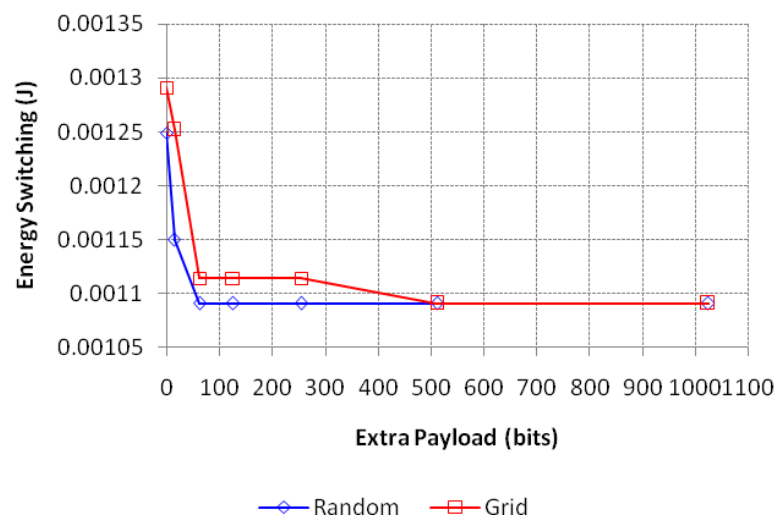

(c)

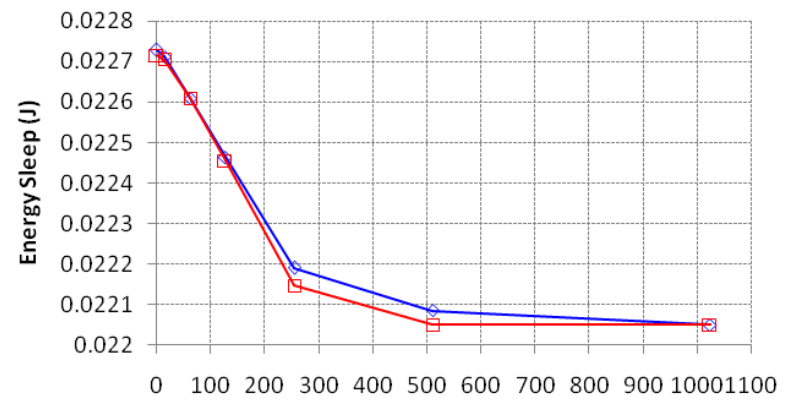

Extra Payload (bits)

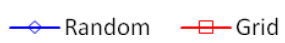

(d)

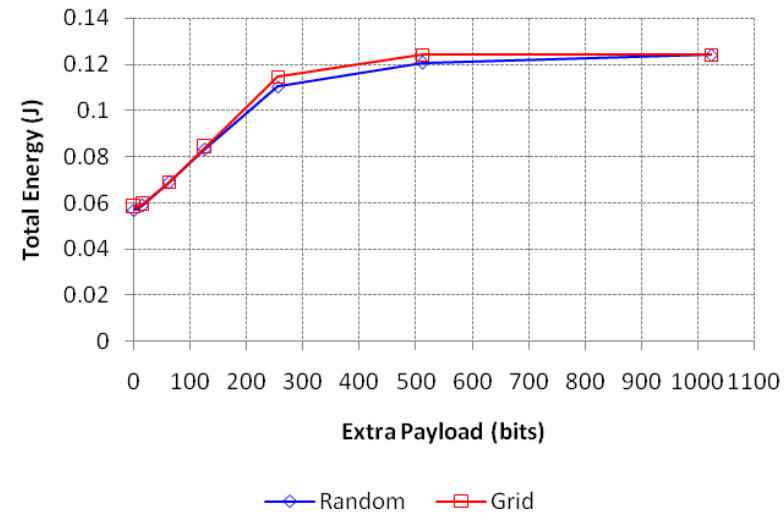

(e)

FIGURE 5: Comparison of random and grid deployment for chipset CC1000 (a) Energy Tx, (b) Energy Rx, (c) Energy Switching, (d) Energy Sleep, (e) Total Energy

Figure in 5 (e) shows the total energy consumption by the chipset $\mathrm{CC} 1000$ for the random and grid deployment. It has been evident from the figure that the energy consumption by the sensor nodes placed in a grid fashion is more than that of the sensor nodes placed in the random deployment.

\section{CASE 5: Energy consumed by chipset $\mathrm{CC1010}$ for random} vs. grid deployment

Figure 6 (a) shows the comparison of energy consumed during transmission of data. The figure clearly depicts that the both the deployment strategies consume same amount of energy for the initial values of extra payload. As the value of extra payload increases, the Grid distribution consumes more energy than that of the random distribution. As the value of Extra payload touches its peak, the random and grid again coincides at a single point showing equal amount of energy consumption.

Figure 6 (b) shows the energy consumed by the sensor node during the reception of the data for random and grid distribution. The energy consumed by the random deployment during reception is almost remains constant for the entire range of values of the extra payload. For the grid distribution, it can be seen that the energy Rx shows a steep increase in the energy 
consumption for a small increment in extra payload and then it drops downs till it reaches the 512 bits of extra payload. After that Energy Rx for grid and random distribution coincides and remains constant thereafter for the increasing vales of extra payload.

Figure 6 (c) shows the comparison of the energy consumed in transition for the random and grid distribution. It is evident from the figure that the senor nodes placed in grid network consumes more energy in transition from one state to another than that of random network.

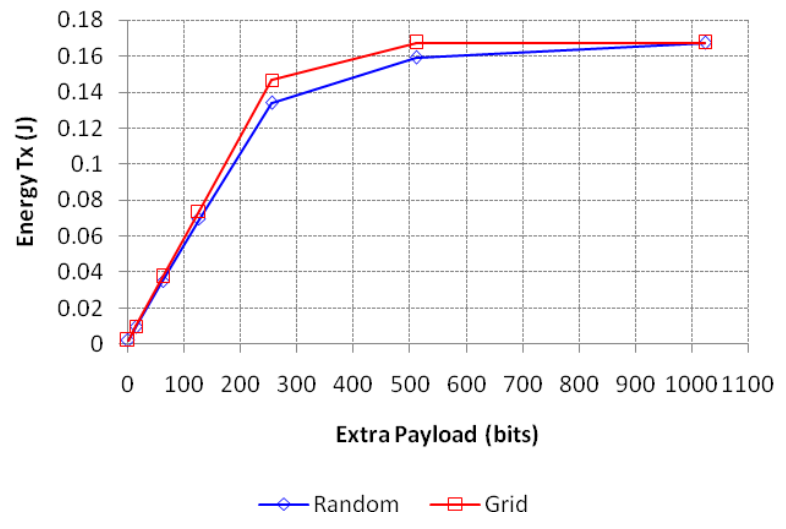

(a)

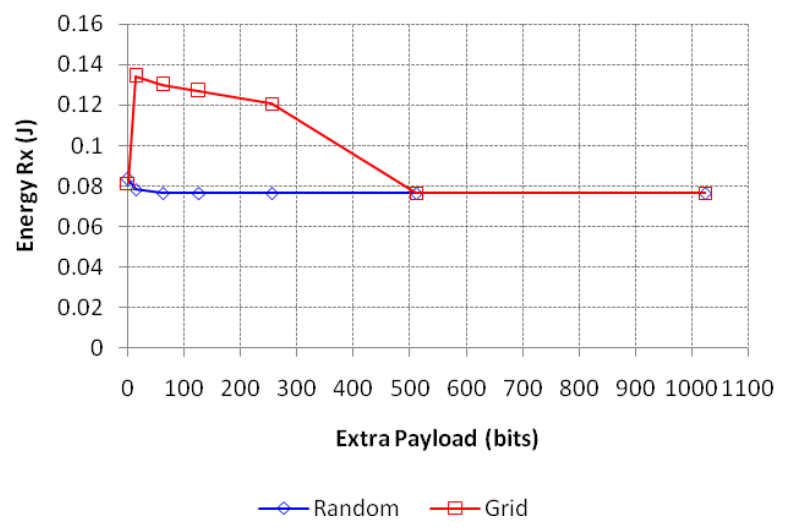

(b)

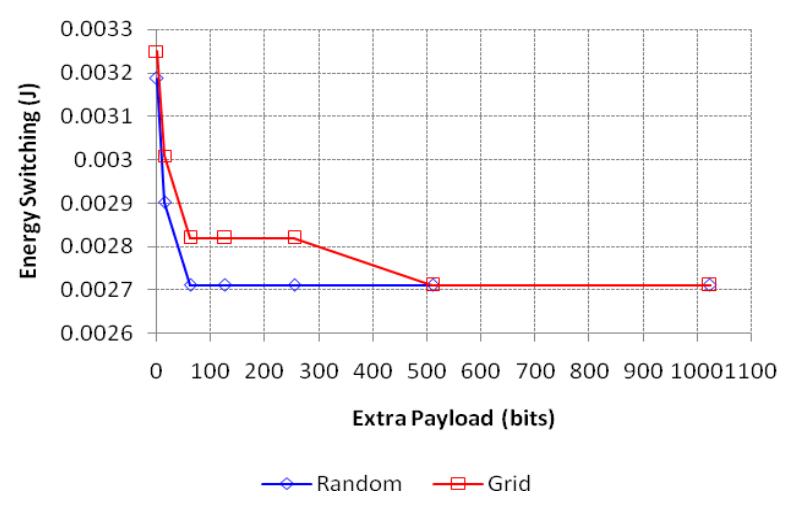

(c)

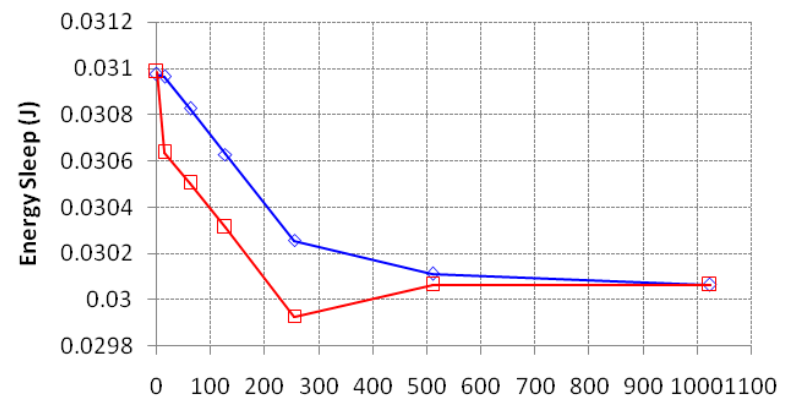

Extra Payload (bits)

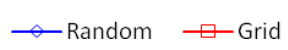

(d)

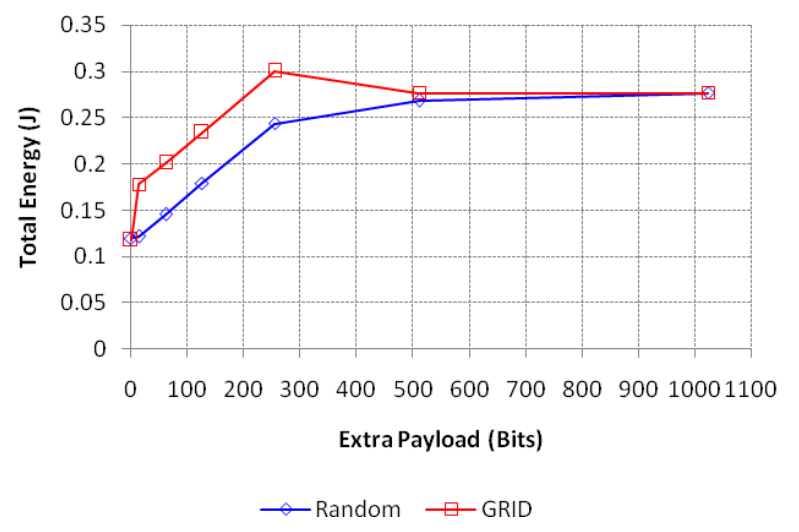

(e)

FIGURE 6: Comparison of random and grid deployment for chipset CC1010 (a) Energy Tx, (b) Energy Rx, (c) Energy Switching, (d) Energy Sleep, (e) Total Energy

For the extra payload of 1 bit, the energy consumed in switching by the node deployed randomly is $0.003187 \mathrm{~J}$ and is $0.003249 \mathrm{~J}$ for the sensor node deployed in grid fashion. Both the deployments shows a steep decrease in the energy consumption upto the extra payload of 63 bits, and then they become stable and finally meets at the point where the value of extra payload is 511 bits, after which they remain constant.

Figure 6 (d) shows the comparison of the energy consumed in sleep for the random and the grid deployment of the sensor networks. It is seen in the figure that more energy is consumed by the random deployment, while grid consumes less energy in sleep mode. Also it is evident from the figure that for the lower value of extra payload, higher is the value of the energy consumption, and it decreases as the value of the extra payload increases. And then at a certain point the energy consumption by both the random and grid distribution becomes equivalent.

Figure 6 (e) shows the cumulative effect of all the energy compositions for the random and grid distribution. Overall it is estimated that the grid deployment of the sensor nodes in the wireless sensor network consumes more energy that the random deployment. 


\section{CONCLUSIONS}

In this paper we discussed the two types of deployments of sensor nodes possible for the wireless sensor networks. We evaluated the energy composition requirement for both the cases for the three chipsets. It has been observed that energy required for data transmission and switching from one state to another by the three chipsets is less when the nodes in the sensor network are deployed randomly. It is also observed that grid deployment consumes lower energy than random deployment during the sleep mode. In case of data reception mode, the chipset TR1001 and CC1000 consumes lower energy when deployed in grid fashion while chipset CC1010 consumes lower energy when deployed in random fashion. Overall it is found that the energy requirement of the grid deployment is more than that of random deployment. Hence it is concluded that random deployment is more energy efficient.

As a future work, we will study few more deployment strategies, and investigate and compare the energy consumption by those new and older deployment strategies.

\section{REFERENCES}

[1] I.F.Akyildiz,W.Su, Y.Sankarasubramaniam, and E. Cayirci. 2002. Wireless sensor networks: A survey. IEEE Communications Magazine, 40(8):102-14.

[2] Sung-Chan Choi, Jang-Won Lee, Yeonsoo Kim, Hakjin Chong. 2007. An Energy-Efficient MAC Protocol with Random Listen-Sleep Schedule for Wireless Sensor Networks. IEEE.

[3] Matthias Ringwald, Kay R*omer. 2007. Deployment of Sensor Networks: Problems and Passive Inspection.

[4] Kenan Xu ,Glen Takahara, Hossam Hassanein, On the robustness of grid-based deployment in wireless sensor networks.

[5] E. S. Biagioni and G. Sasaki. 2003. Wireless Sensor Placement for Reliable and Efficient Data Collection. IEEE HICSS'03, pp. 127b.
[6] Pervasive Computing ETH Zurich, Switzerland H. Poor. 1985. An Introduction to Signal Detection and Estimation. New York: Springer-Verlag.

[7] Andrew Howard, Maja J Matari'c, and Gaurav S Sukhatme. 2002. Mobile Sensor Network Deployment using Potential Fields: A Distributed, Scalable Solution to the AreaCoverage Problem. Submitted to the $6^{\text {th }}$ International Symposium on Distributed Autonomous Robotics System (DARS02).

[8] Ali Iranli, Morteza Maleki, Masooud Pedram. 2005. Energy Efficient Strategies for Deployment of a Two-Level Wireless Sensor Network.

[9] Richard Tynan, Antonio Ruzzelli, G.M.P.O'Hare. 2005. A Methodology for the Deployment of Muti-Agent Systems on Wireless Sensor Networks.

[10] Chih-Yung Chang, Kuei-Ping Shih and Shih-Chieh Lee. 2005. ZBP: A Zone Based Broadcasting Protocol for Wireless Sensor Networks. Wireless Personal Communication 33:53-68.

[11] Jiaghao Li, Yuebin Bai, Haixing Ji, Jihong Ma, Yong Tian and Depei Qian. 2006. POWER: Planning and Deployment Platform for Wireless Sensor Networks. In proceedings of the Fifth International Conference on Grid and Cooperative Computing Workshops (GCCW'06), IEEE.

[12] Chih-Yung Chang, Kuei-Ping Shih, Hsu-Ruey Chang, Hsiao-Jung Liu. 2006. Energy- Balanced Deployment and Topology Control for Wireless Sensor Networks. In IEEE GLOBECOM proceedings.

[13] Pradnya Gajbhiye, Anjali Mahajan. 2008. A Survey of Architecture and Node Deployment in Wireless Sensor Network.

[14] Ian F. Akyildiz, Weilian Su, Yogesh Sankarasubramaniam, and Erdal Cayirci. A survey on Sensor networks. Georgia Institute of Technology

[15] Rabi Mahapatra. Embedded systems for wireless sensor networks. 